\title{
Behavioral momentum and resurgence: Effects of time in extinction and repeated resurgence tests
}

\author{
Mary M. Sweeney • Timothy A. Shahan
}

Published online: 28 August 2013

(C) Psychonomic Society, Inc. 2013

\begin{abstract}
Resurgence is an increase in a previously extinguished operant response that occurs if an alternative reinforcement introduced during extinction is removed. Shahan and Sweeney (2011) developed a quantitative model of resurgence based on behavioral momentum theory that captures existing data well and predicts that resurgence should decrease as time in extinction and exposure to the alternative reinforcement increases. Two experiments tested this prediction. The data from Experiment 1 suggested that without a return to baseline, resurgence decreases with increased exposure to alternative reinforcement and to extinction of the target response. Experiment 2 tested the predictions of the model across two conditions, one with constant alternative reinforcement for five sessions, and the other with alternative reinforcement removed three times. In both conditions, the alternative reinforcement was removed for the final test session. Experiment 2 again demonstrated a decrease in relapse across repeated resurgence tests. Furthermore, comparably little resurgence was observed at the same time point in extinction in the final test, despite dissimilar previous exposures to alternative reinforcement removal. The quantitative model provided a good description of the observed data in both experiments. More broadly, these data suggest that increased exposure to extinction may be a successful strategy to reduce resurgence. The relationship between these data and existing tests of the effect of time in extinction on resurgence is discussed.
\end{abstract}

Keywords Resurgence · Extinction · Behavioral momentum $\cdot$ Relapse $\cdot$ Alternative reinforcement

M. M. Sweeney $(\triangle) \cdot$ T. A. Shahan

Department of Psychology, Utah State University, 2810 Old Main

Hill, Logan, Utah 84322, USA

e-mail: sweeneymm@gmail.com

T. A. Shahan

e-mail: tim.shahan@usu.edu
Resurgence is relapse that occurs following the removal of alternative reinforcement introduced during the extinction of an operant response. Resurgence has practical implications for treatments using alternative reinforcement to reduce problem behaviors, because it suggests that the removal or reduction of alternative reinforcement following treatment can result in an increase in the problem behavior. Many popular behavioral treatments involve alternative reinforcement, such as contingency management for substance abuse (e.g., Higgins et al., 2010; Silverman et al., 2007) and differential reinforcement of alternative behavior (DRA) in individuals with intellectual or developmental disabilities (Petscher, Rey, \& Bailey, 2009). Although treatments that use alternative reinforcement are often effective at reducing problem behavior during treatment, the risk of relapse when alternative reinforcement is reduced or removed has led to a recent revival in basic (Lieving \& Lattal, 2003; Quick, Pyszczynski, Colston, \& Shahan, 2011; Winterbauer \& Bouton, 2010) and applied (Volkert, Lerman, Call, \& Trosclaire-Lasserre, 2009) research on resurgence (see Lattal \& St. Peter Pipkin, 2009, for a review).

Behavioral momentum theory has been useful for understanding the persistence (e.g., Nevin, Mandell, \& Atak, 1983) and relapse (Podlesnik \& Shahan, 2010) of operant behavior - as such, the application of behavioral momentum theory to resurgence could shed light on important determinants of resurgence magnitude. In an effort to integrate resurgence into behavioral momentum theory, Shahan and Sweeney (2011) proposed a quantitative model of resurgence based on the augmented-extinction model (Nevin \& Grace, 2000). The augmented-extinction model suggests that experience with higher rates of reinforcement within a discriminativestimulus context prior to extinction renders an operant response more resistant to the disruptive effects of extinction. The model suggests:

$\log \left(\frac{B_{t}}{B_{0}}\right)=\frac{-t(c+d r)}{r^{b}}$ 
where $B_{t}$ is the response rate at time $t$ in extinction and $B_{0}$ is the baseline response rate before extinction, $c$ is the suppressive effect of breaking the response-reinforcer contingency, $d$ scales the suppression associated with the elimination of reinforcers from the situation (i.e., generalization decrement), $r$ is the rate of reinforcement within the context at baseline, and $b$ is sensitivity to the reinforcement rate. As time in extinction increases, the disruptive impact increases (in the numerator) but is counteracted by previous experience with higher reinforcement rates in the discriminative context (in the denominator). The reinforcement experienced in the context includes all sources of reinforcement, regardless of whether they are contingent upon the target response, independent of the target response, or even contingent on an alternative response. This prediction stems from behavioral momentum theory's suggestion that resistance to disruption is governed by the Pavlovian discriminativestimulus-reinforcer relation, which has been supported by research with species ranging from fish to humans (e.g., Ahearn, Clark, Gardenier, Chung, \& Dube, 2003; Cohen, 1996; Igaki \& Sakagami, 2004; Nevin, Tota, Torquato, \& Shull, 1990; Shahan \& Burke, 2004). Nevin, McLean, and Grace (2001) have shown that the $c$ and $d$ parameters are independent, vary as expected with experimental manipulations, and combine additively, as suggested by the model. Equation 1 also accounts for the partial-reinforcement extinction effect, because at very high rates of reinforcement, the stimulus change associated with removal of the reinforcers from the situation (i.e., generalization decrement - $d r$ ) serves as a larger disruptor than does removal of reinforcers arranged on a schedule of partial reinforcement (Nevin \& Grace, 2005). Equation 1 has provided a successful account of extinction of operant behavior in basic research and in applied settings (Nevin \& Shahan, 2011, for a review).

Shahan and Sweeney (2011) extended Eq. 1 to resurgence by suggesting that alternative reinforcement during extinction of a target behavior has two effects. First, alternative reinforcement further disrupts the target behavior. Second, such reinforcement contributes to the strength of the target behavior by serving as an additional source of reinforcement in the context. Thus, the model suggests:

$\log \left(\frac{B_{t}}{B_{0}}\right)=\frac{-t\left(k R_{a}+c+d r\right)}{\left(r+R_{a}\right)^{b}}$

where all terms are as in Eq. 1. The added variable $R_{a}$ is the rate of alternative reinforcement during extinction, and the added parameter $k$ scales the disruptive impact of the alternative reinforcement during extinction. The inclusion of $k R_{a}$ increases the suppressive impact in the numerator, with higher rates of alternative reinforcement producing more suppression of the target behavior. When alternative reinforcement is removed, $k R_{a}$ is zero, and the target behavior increases as a result of the decrease in disruption. In addition, because $R_{a}$ is included in the denominator, alternative reinforcement experienced in the context during extinction also contributes to the future strength of the target behavior.

Equation 2 describes several known findings in the resurgence literature and fits existing data well (Shahan \& Sweeney, 2011). One such finding is that less resurgence occurs following longer exposure to extinction plus alternative reinforcement (Leitenberg, Rawson, \& Mulick, 1975, Exp. 4). Equation 2 captures the effect of extended exposure to extinction plus alternative reinforcement through its use of time in extinction as a factor that increases the impact of disruption over time. As time in extinction increases, $t$ becomes larger, and consequently the larger numerator predicts that the removal of alternative reinforcement after extended periods of extinction will result in less resurgence.

A related prediction of Eq. 2 is that resurgence should decrease across repeated tests. In other words, when subjects are not returned to baseline contingencies of reinforcement for the target response, $t$ continues to grow as exposure to extinction plus alternative reinforcement increases, and thus the model predicts that resurgence should decrease across each removal of alternative reinforcement. Figure 1 shows a simulation of this prediction using the exponentiated version of Eq. 2, which avoids logarithmic transformation of response rates and permits the inclusion zero values. The exponentiated version is

$\frac{B_{t}}{B_{0}}=10^{\frac{-t\left(k R_{a}+c+d r\right)}{\left(r+R_{a}\right)^{b}}}$

where all terms are as in Eq. 2. This simulation in Fig. 1 is supported by evidence from two studies, Quick et al. (2011) and Wacker et al. (2011). Quick et al. investigated resurgence of cocaine seeking in rats following the removal of alternative food reinforcement for nose pokes during extinction.

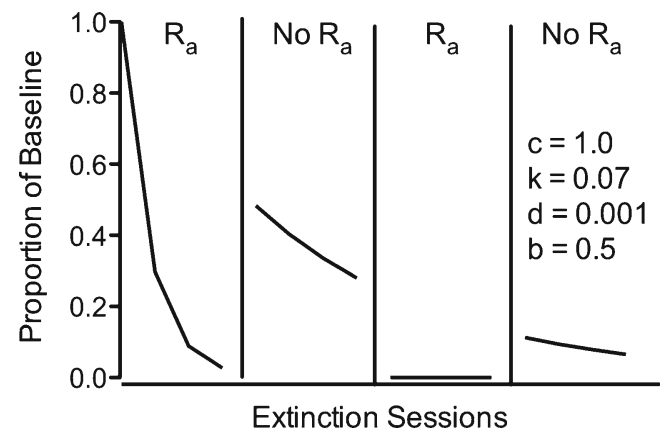

Fig. 1 Simulation produced by Eq. 3 using a baseline reinforcement rate of variable-interval (VI) $60 \mathrm{~s}$ and an alternative-reinforcement rate of VI $30 \mathrm{~s}$ and repeated introductions $\left(R_{a}\right)$ and removals (no $R_{a}$ ) of alternative reinforcement. From "A Model of Resurgence Based on Behavioral Momentum Theory," by T. A. Shahan and M. M. Sweeney, 2011, Journal of the Experimental Analysis of Behavior, 95, p. 98. Copyright 2011 by Wiley Blackwell. Reprinted with permission 
The researchers introduced and removed alternative food reinforcement twice while keeping the extinction of cocaine seeking in place. Relapse during the second resurgence test was significantly less than in the first resurgence test - consistent with the predictions of Eq. 3. In an applied study with children with developmental disabilities, Wacker et al. alternated extinction of the problem behavior with extinction plus alternative reinforcement, in the form of functional communication training (FCT). The resurgence of the problem behavior that occurred following FCT generally decreased with each removal of alternative reinforcement. In fits of Eq. 3 to the data, Wacker et al. found that the model accurately described the decreased resurgence seen following repeated FCT. Although the percentage of variance accounted for was relatively low as compared with fits of the model to data from basic laboratories, the fits were compelling given the variability inherent in the data set collected in children's homes with their mothers serving as therapists.

The purpose of the present experiments was to examine resurgence across repeated tests under conditions explicitly designed to test Eq. 3. Experiment 1 was designed to establish that resurgence decreases across repeated tests with simple foodmaintained behavior, in a manner consistent with the predictions of Eq. 3 and with existing data from more complex situations (Quick et al., 2011; Wacker et al., 2011). Though the data from Quick et al. and Wacker et al. are consistent with the predictions of Eq. 3 displayed in Fig. 1, there are no data comparing a condition with repeated resurgence tests to a condition with constant alternative reinforcement. Equation 3 predicts that not only should resurgence decrease across repeated resurgence tests as time in extinction increases, but resurgence should be comparably low at a given time $t$ in extinction in a condition in which the first removal of alternative reinforcement occurs at time $t$ and a condition that includes alternative-reinforcement lapses prior to time $t$. Experiment 2 tested these predictions.

\section{Experiment 1}

In Experiment 1, we assessed the effects of repeated implementations of extinction plus alternative reinforcement on subsequent resurgence under conditions designed to test the predictions of Eq. 3. The experimental parameters used in Experiment 1 provided the basis for the simulation in Fig. 1.

Method

Subjects Twelve unsexed homing pigeons (Double T Farm, Glenwood, IA) with varied previous experimental histories served as the subjects. The pigeons were maintained at approximately $80 \%$ of their free-feeding weight $( \pm 15 \mathrm{~g})$ via postsession feedings in the home cage and adjustments of the hopper duration across subjects ranging from 1.3 to $2 \mathrm{~s}$. The colony room was on a 12:12-h light:dark cycle with lights on at $0700 \mathrm{~h}$. Experimental sessions occurred in three squads of four pigeons each, with each squad running at approximately the same time each day.

Apparatus The experimental sessions took place in four Lehigh Valley Electronics pigeon operant chambers that measure $350 \mathrm{~mm}$ long, $350 \mathrm{~mm}$ high, and $300 \mathrm{~mm}$ wide. Three response keys, $83 \mathrm{~mm}$ apart, each $25 \mathrm{~mm}$ in diameter, were centered on the front panel of the chamber. The keys were transilluminated via back-mounted in-line projectors and could display yellow, blue, and red homogeneous hues, as well as three separate white shapes (circle, horizontal line, and vertical line) on a black background. About $0.1 \mathrm{~N}$ of force was required to operate the keys. A house light located $76 \mathrm{~mm}$ above the center key provided general illumination directed toward the chamber ceiling. When the hopper was elevated, a miniature bulb illuminated the available Purina Pigeon Chow in a 50 -mm wide $\times 55$-mm tall aperture located $130 \mathrm{~mm}$ below the center key. A fan mounted to the outside of each chamber provided ventilation. The fan and white noise helped to mask extraneous sounds. Med Associates (St. Albans, VT) programming and interfacing were used to control the execution and recording of experimental events.

Procedure Experiment 1 involved three phases: baseline, extinction, and test. Because the subjects had previous experimental histories, no shaping or pretraining was necessary before the baseline phase. Baseline consisted of ten sessions, during which only the center key was illuminated and displayed a white vertical line on a black background. Pecks to the center key (the target response) produced food on a variable-interval (VI) 60-s schedule of reinforcement. When a food delivery was arranged, the next target response turned off the house light and response key and produced access to the illuminated hopper aperture. Following the hopper presentation, the key and the house light were relit, and the VI timer restarted. Sessions were $45 \mathrm{~min}$ long, excluding hopper time.

During extinction (EXT), pecks to the center key (vertical line) no longer produced food, but pecks to the right key (blue hue) produced food on a VI 30-s schedule. As during baseline, hopper time was excluded from the session time, and the only stimulus illuminated during hopper delivery was the food aperture light. EXT lasted for three days.

Next, in the test phase, both the center key and the right key remained illuminated with their respective stimuli, but neither produced food. The test phase lasted for three days. Next, EXT and test were repeated (EXT 2, Test 2) for three days each, without returning to baseline.

\section{Results}

The means and standard deviations of the target key response rate, alternative key response rate, inactive key response rate, 
Table 1 Experiment 1 response rates and food rates

\begin{tabular}{llllll}
\hline & Baseline & EXT & Test & EXT 2 & Test 2 \\
\hline Target & $66.43(28.28)$ & $1.95(1.92)$ & $8.17(7.17)$ & $0.13(0.18)$ & $75.56(31.22)$ \\
Alternative & - & $81.32(37.50)$ & $19.20(9.73)$ & $0.001(0.002)$ & $12.45(4.68)$ \\
Inactive & $0.04(0.07)$ & $0.003(0.01)$ & $0.49(1.38)$ & $1.93(0.03)$ & - \\
Foods & $0.97(0.02)$ & $1.91(0.05)$ & - & $0.35(0.62)$ \\
\hline
\end{tabular}

Displayed are the means and $S D$ s (in parentheses) for the target key response rate, alternative key response rate, and inactive response rates (in pecks/ $\mathrm{min}$ ), as well as the obtained food delivery rate (in foods/min), for each phase of Experiment 1 . For baseline, each pigeon's individual mean for the last five days was first calculated and then included in the analysis above. For each extinction phase, each pigeon's individual mean was calculated for the entire phase and then included in the calculation above. In each cell of the table above, $n=12$

and obtained food delivery rate during each phase of Experiment 1 are presented in Table 1. Acquisition of the target response (i.e., pecks to the vertical line) proceeded normally during baseline. Pecks to the inactive (unlit) response keys were negligible for all pigeons.

The target response rate decreased during the three days of EXT. Acquisition of the alternative response was rapid; pigeons earned close to the maximum food delivery rate on the first day of EXT ( $M=1.88$ foods $/ \mathrm{min}, S D=0.08)$. Responding on the inactive key continued to be negligible in all subjects. The average target response rate on the last day of EXT was 0.08 pecks $/ \mathrm{min}, S D=0.16$.

Figure 2 displays target response rates during the final session of each exposure to the repeated EXT and the first session of each test phase. During the first session of the test phase, target response rates increased relative to the last session of EXT. The increase from the last session of EXT 2 to the first session of Test 2 was smaller than the increase from the last session of EXT to the first session of Test. We conducted a $2 \times 2$ within-subjects repeated measures analysis of variance (ANOVA) with the factors Transition and $R_{a}$. Target response rates for the sessions making up the first transition (i.e., EXT to Test) were coded as part of the first level of transition, whereas target response rates for sessions EXT 2 to Test 2 were coded as

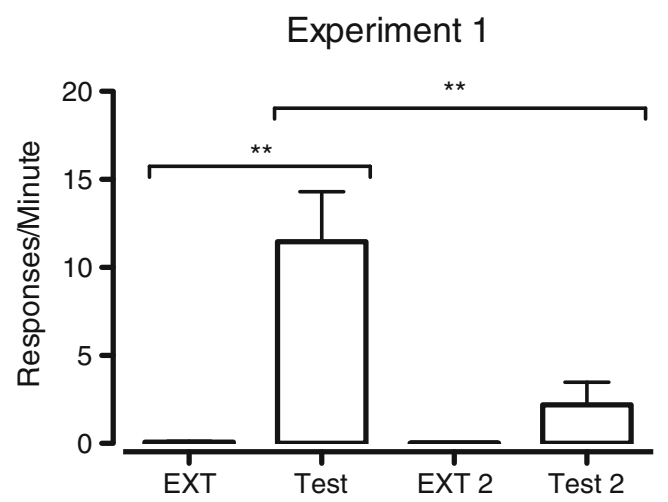

Fig. 2 Comparison of the mean $(n=12)$ target response rates (with $S E \mathbf{s}$ ) on the last day of EXT, the first day of test, the last day of EXT 2, and the first day of Test 2 during Experiment $1 .{ }^{* *} p<.01$ for simple effects part of the second transition. The level of the factor $R_{a}$ was determined by whether alternative reinforcement was present during the session (EXT and EXT 2) or absent (Test and Test 2). We found significant main effects of transition $[F(1,11)=$ $11.98, p<.01]$ and of $R_{a}[F(1,11)=15.15, p<.01]$, as well as a Transition $\times R_{a}$ interaction $[F(1,11)=11.85, p<.01$.], capturing that the effect of removing alternative reinforcement on target response rates was different from the first to the second resurgence test. In simple-effects analyses, we found a statistically significant increase in target response rate on the first day of Test relative to the last day of $\operatorname{EXT}[F(1,11)=$ $16.24, p<.01]$, and also a significant decrease in target response rate on the first day of Test 2 relative to the first day of Test $[F(1,11)=11.92, p<.01]$. No significant difference emerged between target response rates on the last day of EXT and the last day of EXT $2[F(1,11)=2.55, p=.14]$, nor a significant difference between the last day of EXT 2 and the first day of Test $2[F(1,11)=2.85, p=.12]$.

Given the visual increase from the last day of EXT 2 to the first day of Test 2, the data were examined for consistent patterns at the individual-subject level. Figure 3 displays the transitions for Test and Test 2. It is clear that for the first test, all but one subject showed an increased target response rate when alternative reinforcement was removed. For Test 2, only two subjects showed notable increases in target response rate and were driving the visual difference between mean target response rate on the last day of EXT 2 and the first day of Test 2 .

Equation 3 was fitted to the mean subject data across all sessions, which is displayed in Fig. 4. As in Shahan and Sweeney (2011), the $d$ parameter was fixed to a value of $0.001, b$ was fixed to 0.5 , and the values of the variables $t, R_{a}$, and $r$ were determined from the experimental parameters of time in extinction, alternative-reinforcement rate, and baseline reinforcement rate, respectively. Because the design included no return to baseline conditions, $t$ increased daily by a value of 1. During EXT and EXT 2, the value of $R_{a}$ in the numerator was set to 120 (i.e., foods per hour), and during Test and Test $2, R_{a}$ in the numerator was set to 0 because alternative reinforcement as a disruptor was not present. Consistent with the usual treatment of the previously experienced response-strengthening effects of 

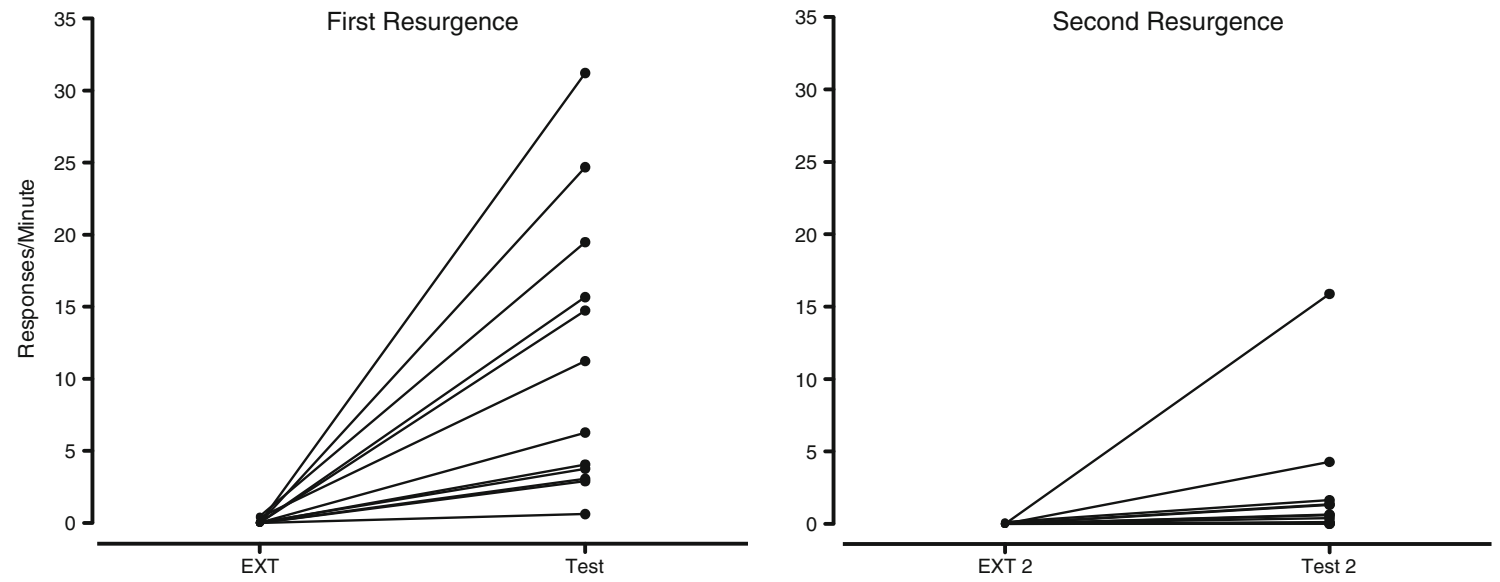

Fig. 3 Individual subject data comparing increases from the first and second resurgence tests in Experiment 1

reinforcement in the denominator of the augmented model during extinction (i.e., $r$ in Eq. 1; see Nevin et al., 2001), the value of $R_{a}$ in the denominator was 120 throughout all EXT and test phases. Only the parameters $k$ and $c$ were free to vary. The least squares regression fit of Eq. 3 to the mean subject data accounted for $99 \%$ of the variance, with $c=2.56$ and $k=0.09$. Table 2 shows the parameter estimates obtained in the fits of Eq. 3 to the individual pigeon data. The median of the individual $R^{2}$ values was $.99(M=.98, S D=.02)$. The median value of parameter $c$ was $2.86(M=2.90, S D=0.99)$, and the median value of $k$ was $0.10(M=0.11, S D=0.04)$.

\section{Discussion}

As in previous experiments in more complex situations (Quick et al., 2011; Wacker et al., 2011), the present experiment showed that resurgence appears to decrease across repeated tests. One could argue that for all but two subjects, resurgence did not occur upon the second removal of alternative reinforcement. Furthermore, the quality of the least squares regression fit to the data from Experiment 1 suggests that, on average, Eq. 3 adequately describes the repeated-resurgence phenomenon, although

\section{Experiment 1}

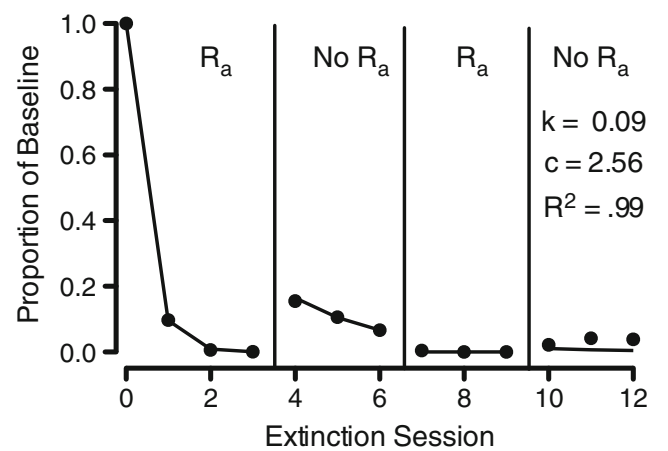

Fig. 4 Least squares regression fit (solid line) of Eq. 3 to the data obtained (filled circles) in Experiment 1 considerable variability occurred in the individual parameter estimates. It is important to note that decreased resurgence across tests is not contradictory to previous findings that repeated examinations of resurgence within subjects result in similar relapses (da Silva, Maxwell, \& Lattal, 2008; Lieving \& Lattal, 2003), because in these previous experiments baseline responding was reestablished before the second examination of extinction and resurgence. In these cases, Eq. 3 requires that the value of $t$ be reset to zero following each baseline, and as such, the model would predict similar resurgence across repeated tests rather than reduced resurgence.

The results of Experiment 1 are also consistent with data from Leitenberg et al. (1975, Exp. 4) in which groups that experienced lengthier extinction plus alternative reinforcement showed less resurgence than did a group that experienced only three sessions of extinction plus alternative reinforcement. On the other hand, a recent failure to replicate the findings of Leitenberg et al. was reported by Winterbauer, Lucke, and Bouton (2013). This discrepancy will be addressed in the General Discussion.

\section{Experiment 2}

The data from Experiment 1 supported the model prediction that resurgence should decrease with repeated resurgence tests. Equation 3 also predicted that resurgence should be similar at time $t$ in extinction in a condition with no previous lapses in alternative reinforcement and at $t$ in a condition with previous removals of alternative reinforcement. Because no data exist that speak to this prediction, Experiment 2 was designed to assess it. As such, in Experiment 2 we compared target responding on the sixth session of extinction across two conditions. In one condition, alternative reinforcement was removed at Sessions 2, 4, and 6 of extinction. In the second condition, the alternative reinforcement was removed only during Session 6 of extinction. A model simulation of 
Table 2 Equation 3 individual subject parameter values for the Experiment 1 fits

\begin{tabular}{lllllllllllll}
\hline Subject & 936 & 966 & 956 & 957 & 8405 & 7095 & 262 & 257 & 175 & 52 & 239 & 887 \\
\hline$k$ & .04 & .10 & .14 & .08 & .14 & .06 & .11 & .16 & .17 & .10 & .06 & .10 \\
$c$ & 1.23 & 3.82 & 1.72 & 3.06 & 3.94 & 4.53 & 1.95 & 2.41 & 2.66 & 3.52 & 3.46 & 2.53 \\
$R^{2}$ & .93 & .99 & .94 & .99 & .99 & .99 & .95 & .99 & .99 & .99 & .99 & .98 \\
\hline
\end{tabular}

Displayed are the values of parameters $k$ and $c$ and the variance accounted for $\left(R^{2}\right)$ for the fits of Eq. 3 to the individual subject data in Experiment 1 . The value of $d$ was fixed to .001 and $b$ to .5 for all fits reported

Experiment 2, using the values of $c$ and $k$ obtained in the fit to the data from Experiment 1, is displayed in Fig. 5.

Method

Subjects The 12 pigeons from Experiment 1, under the same feeding and living conditions, also served as subjects in Experiment 2.

Apparatus Experiment 2 took place in the same operant chambers as Experiment 1. The session start times were approximately equal to those in Experiment 1, with each pigeon running in the same group of four and in the same chamber as in Experiment 1.

Procedure In Experiment 2, we compared resurgence across two conditions following equal baselines. In one condition (Constant- $R_{a}$ ), the alternative reinforcement was constant for five days of extinction of the target response and was removed for the sixth extinction session. The other condition (On/Off$R_{a}$ ) alternated between one day of reinforcement for the alternative response and one day of extinction of the alternative response. Therefore, on Days 2 and 4 of extinction, alternative reinforcement was available for the Constant $-R_{a}$ condition

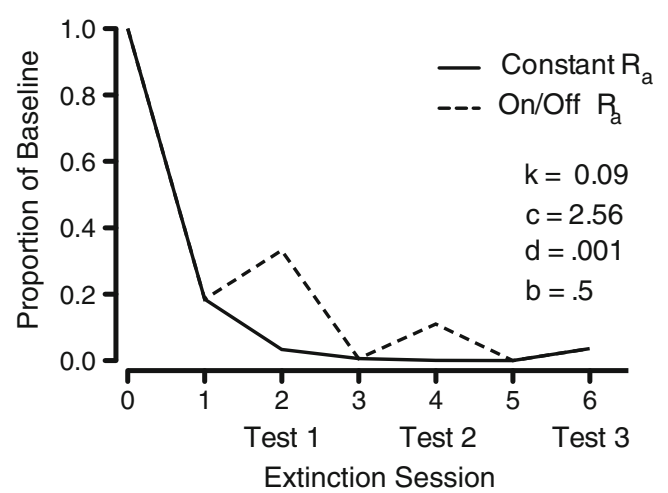

Fig. 5 Simulation of the predictions of Eq. 3 that compares the two conditions in Experiment 2. One condition receives constant alternative reinforcement for the first five extinction sessions (Constant $R_{a}$ ), and the other condition receives alternative reinforcement only on Days 1 , 3 , and 5 of extinction $\left(\mathrm{On} / \mathrm{Off} R_{a}\right)$. Neither condition received alternative reinforcement during Day 6 of extinction. Free parameter values $(k$ and $c$ ) were fixed to the values obtained in the fit to Experiment 1 and unavailable for the On/Off- $R_{a}$ condition. On Day 6 of extinction, alternative reinforcement was unavailable in both conditions. Exposure to extinction of the target response was equal (six days) in both conditions. All 12 subjects experienced both series of Constant- $R_{a}$ and On/Off- $R_{a}$ conditions in a counterbalanced order. Exposure to the second series of conditions was preceded by a return to baseline. Baseline consisted of nine 35-min sessions during which pecks to the center key produced food on a VI 60-s schedule. During the first series of conditions, in which half of the subjects experienced constant $R_{a}$ and half experienced On/Off $R_{a}$, the center key (target response) was illuminated with a yellow homogeneous hue, and the left key (alternative response) was illuminated with a white circle on a black background. In the second series, when the conditions experienced during extinction were reversed for each subject, the center key (target response) was a white horizontal line on a black background and the right key (alternative response) was a red homogeneous hue. During both the first and second baselines, the alternative response key was dark. During both extinction conditions, the alternative response key remained on, regardless of the availability of alternative reinforcement.

\section{Results}

Acquisition of the target response proceeded normally during both series of Experiment 2. During the last five days of baseline, mean target response rates did not differ between Series $1(M=61.85$ pecks $/ \mathrm{min}, S D=27.53)$ and $2(M=$ 61.99 pecks $/ \mathrm{min}, S D=35.02$ ) of the study. Performance in the Constant $-R_{a}$ and the On/Off- $R_{a}$ conditions did not depend on whether the subjects experienced the condition in the first or the second series of Experiment 2. The means and standard deviations of target-key response rate, alternative-key response rate, inactive-key response rate, and obtained food rate across conditions for each day of extinction are presented in Table 3.

Sessions 2, 4, and 6 of extinction (during which alternative reinforcement was removed for at least one condition) will be referred to as Test 1 , Test 2 , and Test 3 , respectively. The data from Test 1 , Test 2 , and Test 3 across conditions are displayed in Fig. 6. Resurgence in the On/Off- $R_{a}$ condition decreased across Tests 1-3, and resurgence during Test 3 did not differ for the Constant $-R_{a}$ and On/Off- $R_{a}$ conditions. A within-subjects 
Table 3 Extinction performance during Experiment 2

\begin{tabular}{|c|c|c|c|c|c|c|}
\hline & Day 1 & Day 2 (Test 1$)$ & Day 3 & Day 4 (Test 2) & Day 5 & Day 6 (Test 3 ) \\
\hline \multicolumn{7}{|l|}{ Constant $R_{a}$} \\
\hline Target & $10.29(8.63)$ & $0.82(0.97)$ & $0.74(0.87)$ & $0.29(0.39)$ & $0.26(0.28)$ & $1.99(2.05)$ \\
\hline Alternative & $63.78(30.97)$ & $72.31(35.50)$ & $72.84(34.17)$ & $72.34(34.38)$ & $65.94(34.03)$ & $29.94(20.71)$ \\
\hline Inactive & $0.005(0.01)$ & $0.009(0.03)$ & $0.01(0.05)$ & $0.06(0.21)$ & $0.96(3.32)$ & $0.73(1.63)$ \\
\hline Foods & $0.88(0.16)$ & $0.96(0.05)$ & $0.96(0.04)$ & $0.96(0.03)$ & $0.95(0.06)$ & - \\
\hline \multicolumn{7}{|l|}{ On/Off $R_{a}$} \\
\hline Target & $4.59(4.73)$ & $11.22(9.50)$ & $3.11(3.13)$ & 4.13 (3.97) & $0.55(0.64)$ & $2.01(2.22)$ \\
\hline Alternative & 77.75 (27.67) & $21.03(10.29)$ & $67.51(23.48)$ & $28.51(16.11)$ & $70.14(26.84)$ & $18.08(12.98)$ \\
\hline Inactive & $0(0)$ & $0.03(0.07)$ & $0.18(0.57)$ & $0.15(0.35)$ & $0.002(0.01)$ & $0.34(1.12)$ \\
\hline Foods & $0.95(0.06)$ & - & $0.95(0.05)$ & - & $0.95(0.05)$ & - \\
\hline
\end{tabular}

Displayed are the means and $S D$ s (in parentheses) for target-key response rate, alternative-key response rate, and inactive response rates (in pecks/ min), as well as for the obtained food delivery rate (in foods/min), for each day of extinction in Experiment 2. Each pigeon's individual rate was included in the calculation above. In each cell of the table above, $n=12$. Days 2, 4, and 6 represent the test days, during which no alternative reinforcement was available for at least one condition

ANOVA using two factors (Condition and Test) was used to compare response rates during Test 1 , Test 2 , and Test 3 across conditions (constant $R_{a}$ vs. On/Off $R_{a}$ ). In this test, we found main effects of condition $[F(1,11)=12.72, p<.01]$ and test $[F(2,22)=9.72, p<.01]$, as well as a Condition $\times$ Test interaction $[F(2,22)=12.17, p<.01]$. Simple-effects comparisons were used to examine the differences between conditions at each test (constant $R_{a}$ v. On/Off $R_{a}$ for Tests 1, 2, and 3), the differences across tests within the On/Off- $R_{a}$ condition (On/Off- $R_{a}$ Test 1 vs. On/Off- $R_{a}$ Test 2, and On/Off- $R_{a}$ Test 2 vs. On/Off- $R_{a}$ Test 3). A significant effect of condition emerged at Test $1[F(1,11)=13.75, p<.01]$ and at Test 2 $[F(1,11)=10.51, p<.01]$. We also observed significant differences in the On/Off- $R_{a}$ condition between Test 1 and Test $2[F(1,11)=12.15, p<.01]$, as well as between Test 2 and Test $3[F(1,11)=5.46, p<.05]$. The results of these comparisons can be seen in Fig. 6. As in Experiment 1, the data were examined on the individual-subject level, and the patterns in general reflected the condition means (see Fig. 7).

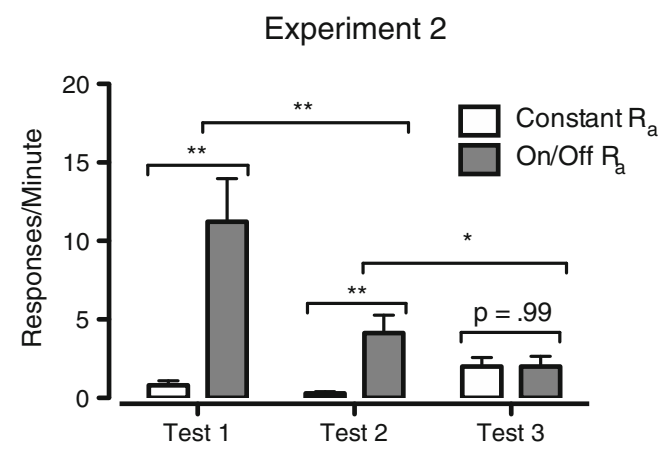

Fig. 6 Comparison of mean target response rates (with $S E$ s) across conditions on Day 2 of extinction (Test 1), Day 4 of extinction (Test 2), and Day 6 of extinction (Test 3 ) in Experiment 2. For each column, $n=$ 12. ${ }^{*} p<.05,{ }^{* *} p<.01$ for simple effects

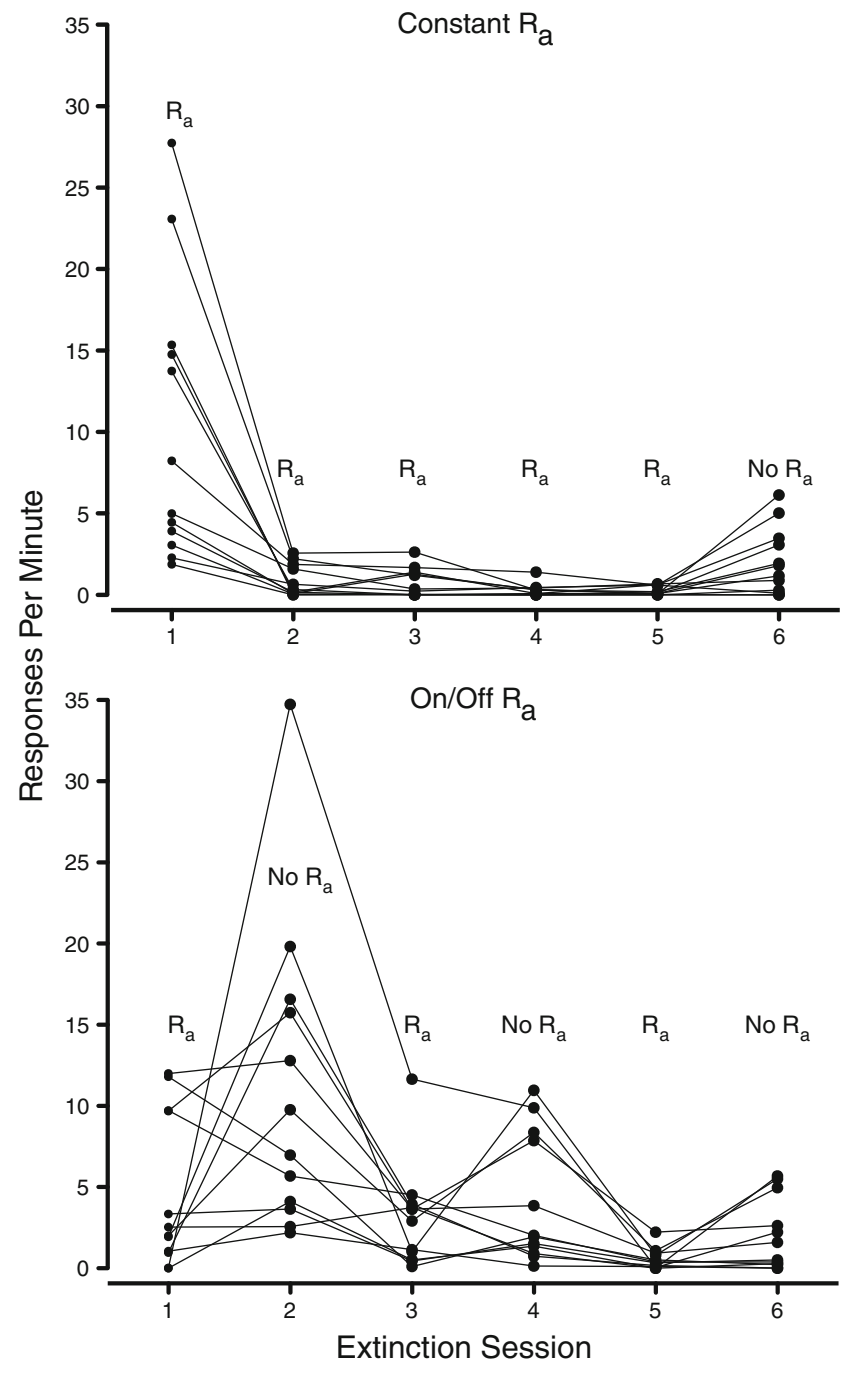

Fig. 7 Individual subject data for target responses in both conditions of Experiment 2. Each line represents one subject, and each condition was experienced by all 12 subjects in counterbalanced order 


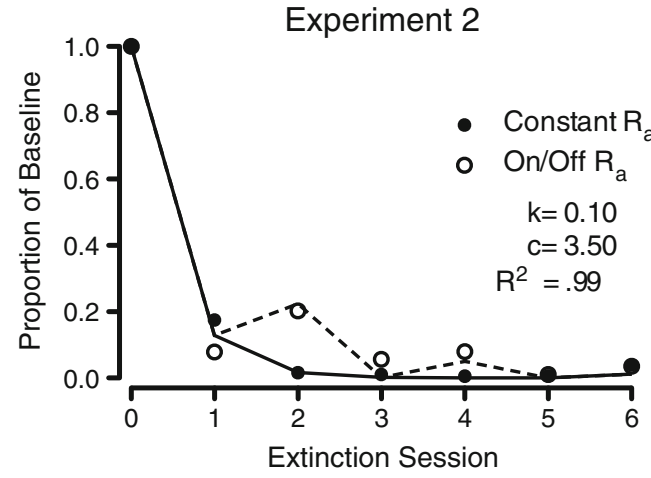

Fig. 8 Least squares regression fits of Eq. 3 to the data obtained in Experiment 2. The solid line represents the fit to the Constant- $R_{a}$ condition, whereas the dashed line fits the On/Off- $R_{a}$ condition. Filled circles (Constant $R_{a}$ ) and open circles (On/Off $R_{a}$ ) illustrate the obtained data

Figure 8 shows a least squares regression fit of Eq. 3 to the data from Experiment 2. As in Experiment 1, the parameter $d$ was fixed to a value of $0.001, b$ was fixed to 0.5 , and the values of $t, R_{a}$, and $r$ were determined from the experimental conditions. The value of $t$ increased by 1 each day that extinction of the target response was in effect, and was reset to 0 following the return to baseline. Whenever alternative reinforcement was available, the value of $R_{a}$ in the numerator was set to 60 (i.e., foods per hour). Whenever alternative reinforcement was unavailable, $R_{a}$ in the numerator was set to 0 . The value of $R_{a}$ in the denominator, however, was 60 throughout extinction in both conditions. It is important to note that in our fits of Eq. 3 to the data from Experiment 2, the value of $R_{a}$ in the denominator is the programmed reinforcement rate of alternative reinforcement when alternative reinforcement was present, rather than the average alternative-reinforcement rate including (for the On/Off- $R_{a}$ condition) zero values for test sessions. This is consistent with previous treatments of baseline reinforcement $(r)$ in the denominator, because this parameter does not decrease across extinction sessions, as more and more zero values of no reinforcement would have to be included in the average. Again, only parameters $k$ and $c$ were free to vary. The least squares regression fit of Eq. 3 to the data accounted for $99 \%$ of the variance $\left(R^{2}=.99\right)$, with $c=3.50$ and $k=0.10$. Although the values of $c$ and $k$ changed from Experiment 1 to Experiment 2, these changes were not large enough to merit concern. When the values of parameters $c$ and $k$ were fixed to the values obtained in the fit to the data from Experiment 1, the variance accounted for by the model decreased only $1 \%\left(R^{2}=.98\right)$. Table 4 displays the obtained parameter values when Eq. 3 was fit to the individual pigeon data. The median $R^{2}$ value for the individual fits was .99 $(M=.98 . S D=.02)$, the median value of parameter $c$ was $4.52(M=4.39, S D=1.89)$, and the median value of $k$ was $.08(M=.11, S D=.06)$.

\section{Discussion}

Two findings of Experiment 2 are key to our present characterization of resurgence: specifically, the suggested roles of time in extinction and exposure to alternative reinforcement. First, in the On/Off $R_{a}$ condition, resurgence decreased across repeated resurgence tests in a manner consistent with the predictions of Eq. 3 and with existing data. Second, target response rates at Test 3 were comparably low for the Constant $-R_{a}$ and On/Off- $R_{a}$ conditions. One consideration is that target response rates at Test 3 were overall very low, and one possibility is that our failure to reject the null hypothesis was inevitable because of low response rates. However, the presence of comparably little resurgence supports our hypothesis rather than challenges it. If previous lapses in alternative reinforcement were responsible for decreased resurgence rather than increased time in extinction, then target response rates on Test 3 for Constant- $R_{a}$ would be comparable to Test 1 for On/Off- $R_{a}$ - this was clearly not the case in our data.

Despite the fact that target response rates at Test 3 were comparable across conditions, the rate of the alternative response at Test 3 was not. As is shown in Table 3, the alternative response rate was considerably lower in the On/Off- $R_{a}$ condition than in the Constant $R_{a}$ condition. The discrepancy between alternative response rate persistence across conditions is consistent with the general behavioralmomentum-based approach of Eq. 3. According to behavioral momentum theory, the decreased rate of alternative reinforcement in the On/Off- $R_{a}$ condition during extinction (if extinction were characterized as baseline for the alternative response) relative to the Constant- $R_{a}$ condition ought to make the alternative response in the On/Off- $R_{a}$ condition less

Table 4 Equation 3 individual subject parameter values for the Experiment 2 fits

\begin{tabular}{lllllllllllll}
\hline Subject & 936 & 966 & 956 & 957 & 8405 & 7095 & 262 & 257 & 175 & 52 & 239 & 887 \\
\hline$k$ & .05 & .14 & .19 & .06 & .25 & .07 & .07 & .05 & .09 & .16 & .12 \\
$c$ & 2.05 & 1.61 & 4.11 & 5.35 & 2.42 & 4.18 & 5.07 & 7.71 & 5.81 & 4.86 & 6.65 & 2.86 \\
$R^{2}$ & .97 & .92 & .99 & .99 & .99 & .99 & .99 & .99 & .99 & .99 & .98 & .94 \\
\hline
\end{tabular}

Values of parameters $k$ and $c$ and the variance accounted for $\left(R^{2}\right)$ for the fits of Eq. 3 to individual subject data in Experiment 2. The value of $d$ was fixed to .001 and $b$ to .5 for all fits reported 
resistant to extinction. The finding that increased exposure to alternative reinforcement seemed to increase the persistence of the alternative response might carry implications for resurgence in applied settings. In these cases, the target response might be an operant problem behavior, and the alternative a socially appropriate response. Given equal instances of problem behavior in a resurgence test, conditions that foster greater persistence of the alternative response might be preferred over those that do not.

\section{General discussion}

The results of both Experiments 1 and 2 suggest that resurgence decreases as time in extinction increases. This finding is consistent with the predictions of Eq. 3, as well as with existing data in which resurgence was repeatedly tested without a return to baseline (i.e., Quick et al., 2011; Wacker et al., 2011). These data and the quantitative framework are also consistent with results from Leitenberg et al. (1975, Exp. 4), which compared resurgence across groups that received three, nine, and 27 sessions of extinction plus alternative reinforcement. The group that received only three sessions of alternative reinforcement showed visually the greatest relapse (although resurgence was not statistically different from the group that received nine days of alternative reinforcement), whereas the group that received 27 sessions of extinction plus alternative reinforcement showed no significant resurgence. Some recent data, however, are challenging for the effect of length of extinction on subsequent resurgence across groups. Winterbauer et al. (2013, Exp. 2) compared groups that received four, 12 , or 36 sessions of extinction plus alternative reinforcement and found no significant differences in subsequent resurgence. The authors suggested that the discrepancy between their results and those of Leitenberg et al. might be the result of a longer baseline in their experiment (12 sessions) relative to the five-session baseline used by Leitenberg et al. However, the equivalent resurgence in their experiment was likely not solely the result of a lengthier baseline, given that decreases in resurgence were seen across repeated tests in Quick et al. (2011), in which rats received between 20 and 25 sessions of baseline cocaine self-administration. Furthermore, decreases in resurgence across implementations and removals of FCT in Wacker et al. (2011) were seen for target problem behavior with an unknown but presumably extensive history of reinforcement.

Winterbauer et al. (2013) also suggested that the predictable, high rate of alternative reinforcement provided on an fixed-ratio (FR) 10 schedule may have contributed to easy discrimination (and consequently similar resurgence) when alternative reinforcement was removed. This interpretation is consistent with their context-change hypothesis. The contextchange hypothesis proposes that resurgence occurs when the organism fails to generalize the learning of the extinguished contingency from the treatment context to the new context in which alternative reinforcement is unavailable. From this perspective, it makes sense that one might observe no differences as a function of length of extinction plus alternative reinforcement, because longer treatment alone does not necessarily mean that what is learned during extinction is more easily generalized to a different context. Our findings from Experiment 1, as well as the data from Quick et al. (2011) and Wacker et al. (2011), are quite consistent with the context-change hypothesis. In these instances, reduced resurgence might be observed because each resurgence test served as generalization training in that extinction was experienced in the context of alternative-reinforcement absence. Our results from Experiment 2, on the other hand, challenge this account of resurgence. If the reduced resurgence observed at Test 3 for the On/Off- $R_{a}$ condition were the result of the subjects' previous exposure to the context without alternative reinforcement, the context-change hypothesis would predict that resurgence for the Constant- $R_{a}$ condition at Test 3 should be equal to resurgence at Test 1 for the On/Off- $R_{a}$ condition. Because we observed comparably low target response rates for both conditions, time in extinction does appear to play an important part in reduced resurgence. Key variables in the behavioral momentum-based model of resurgence might explain why time in extinction was critical in our data and Leitenberg et al. (1975), but showed little impact in Experiment 2 of Winterbauer et al.'s study. A close look at the data reveals a potential source of the discrepancy: The mean alternative-reinforcement rates during extinction in the Winterbauer et al. study varied as a function of group.

Although all subjects in Winterbauer et al. (2013) experienced FR 10 schedules of reinforcement for the alternative response during treatment, treatment ended for the shorter treatment groups at earlier points in alternative response acquisition, meaning that the average alternative response rate across all of treatment was different across groups. The alternative response rate does not enter into the predictions of Eq. 3, but the alternative-reinforcement rate plays a crucial role in the degree of predicted resurgence. Because Winterbauer et al. used an FR schedule, the alternativereinforcement rate directly depended on the rate of the alternative response. In our experimental preparations with VI schedules (in which programmed reinforcement rates are tolerant to fairly wide variations in alternative response rates), we used the programmed rate of alternative reinforcement for sessions in which alternative reinforcement is present as $R_{a}$ in the denominator to predict degree of relapse. When alternative-reinforcement rate is directly tied to alternative response rate, and alternative-reinforcement rate changes considerably as extinction progresses, it may be better to consider the mean alternative-reinforcement rate for the entire phase rather than one programmed rate. 
Although the mean obtained alternative-reinforcement rates across all treatments were not reported in Winterbauer et al. (2013), we estimated alternative response rates (and consequent reinforcement rates because of the ratio schedule) using the GraphClick 3.0 data extraction software. These data suggest that, on average across all sessions of extinction plus alternative reinforcement, the group that received four sessions of extinction received approximately 3.41 alternative food deliveries $/ \mathrm{min}$, the 12 -session group received $3.92 / \mathrm{min}$, and the 36-session group received 5.30/min during extinction. Therefore, although exposure to extinction was greater in the 12- and 36-session groups, the alternative-reinforcement rate was also greater. Higher alternative-reinforcement rates should produce greater resurgence, but longer exposures to extinction should reduce it. These two contributing factors may have acted in opposition, ultimately leading to no difference in resurgence, despite dissimilar exposures to extinction. Leitenberg et al. (1975) did not report either on their obtained reinforcement rate during extinction or on the reinforcement schedule type used for the alternative response, but if no differences in reinforcement rates occurred during extinction across groups, one would only expect differences in observed resurgence across their groups to be a function of time in extinction. Even so, this discrepancy does point out the need for a better understanding of factors that may interact with the effects of extended alternative-reinforcement training on subsequent resurgence.

The variable rates of alternative reinforcement accompanying fixed-ratio schedules also points out the need for a better understanding of how to incorporate reinforcement rates from more dynamic environments into resurgence predictions. In other words, it remains to be seen which is more important in terms of the stimulus-reinforcer relationship, the most recently experienced rate of reinforcement, initially experienced rates, or some average of reinforcement rates experienced in the context. Experiments explicitly designed to test such effects are needed. Furthermore, it remains possible that reduced resurgence following greater exposure to extinction may be limited to within-subjects comparisons and may not be consistently seen across groups. Finally, a related potential limitation of the present experiments is the use of experienced rather than naïve subjects. Resurgence is not thought to decrease if baseline training is reimplemented (Lieving \& Lattal, 2003), but future studies that are designed to directly examine the effect of prior extinction on subsequent persistence (and consequent behavioral momentum model parameter estimates) is merited.

From the perspective of our model, the effects of time in extinction and exposure to alternative reinforcement on resurgence are linked. The disruptive impact of alternative reinforcement grows larger as time in extinction increases - in Eq. 3, this is captured by the multiplicative effect of $t$ on the numerator. Consequently, the decrease in disruption from removing alternative reinforcement during the resurgence test has less of an impact when the time in extinction is lengthy. Therefore, the effects of increased exposure to alternative reinforcement and increased time in extinction on resurgence are not easily disentangled in model predictions. However, the results of Experiment 2 may suggest a more important role for extinction of the target response than for exposure to alternative reinforcement in reducing resurgence. This is because at Test 3 , the times in extinction were equal in both conditions $(t=6)$, but exposures to the alternative reinforcement were different. That is, the pigeons in the Constant $-R_{a}$ condition had experienced five sessions of alternative reinforcement, and those in the On/Off- $R_{a}$ condition had only experienced three sessions of alternative reinforcement. Although the model in its present form does not make predictions that pit exposure to alternative reinforcement versus time in extinction, research that assesses the relative impacts of these two factors on reduced resurgence could prove useful.

The relative contributions of alternative reinforcement and time in extinction are important to consider, because without alternative reinforcement during extinction, there is no increased risk of relapse resulting from removal of the alternative reinforcement upon completion of the treatment (not considering risk for relapse from other sources such as contextual renewal and reinstatement). Because alternative reinforcement increases the stimulus-reinforcer relationship of the context, it increases resistance to change despite its disruptive impact (see Nevin \& Shahan, 2011, for discussion). For example, Mace et al. (2010, Exp. 1) implemented alternative reinforcement in the treatment of problem behavior in three participants with developmental disabilities. Although the presence of alternative reinforcement in the same context as baseline reinforcement for the problem behavior generally reduced problem behavior when it was in place, problem behavior was more persistent during subsequent extinction than when extinction followed a baseline condition without alternative reinforcement. An important procedural difference between our animal model and Mace et al.'s Experiment 1 is that they did not include extinction of the problem behavior alongside alternative reinforcement. Still, their data illustrate that alterative reinforcement, although disruptive, represents an increased risk of later persistence. Again, future work should directly test the importance of increased exposure to alternative reinforcement relative to longer time in extinction, as both factors may contribute to decreased resurgence.

This study was designed to examine the effect of time in extinction on repeated tests. The quantitative model of resurgence predicted that without a return to baseline, relapse following the removal of alternative reinforcement should have decreased with each resurgence test. In Eq. 3, this is manifest by larger values of $t$ rendering the impact of removing $R_{a}$ less influential. The obtained data were consistent with this prediction in Experiment 1, which demonstrated that the 
resurgence of simple, food-maintained behavior decreased across repeated resurgence tests, in a manner consistent with existing cocaine self-administration and applied behavioral studies. Experiment 2 further challenged the quantitative model of resurgence by comparing resurgence at equal time points in extinction across two conditions, one with previous resurgence tests and one that was tested for the first time. Target response rates were equally low across conditions at the same time point in extinction, despite dissimilar exposure to the alternative reinforcement, consistent with the predictions of Eq. 3. The qualitative prediction of decreased resurgence with increased time in extinction was well met by the data, but we observed considerable variability in the parameter estimates within subjects (across experiments) and between subjects (within experiments). Despite the variability in parameter estimates, currently there is no comparable model against which we might judge our fits of extinction and resurgence. Overall, these data and their consistency with translational work are promising for the general approach of the quantitative model of resurgence. If our results continue to generalize, further work in this vein might be useful for reducing the resurgence of problem behaviors and encouraging the persistence of desirable behaviors in applied settings.

Author note The authors thank Meredith Berry, Kelli Colston, Laura Hernandez, Daniel Hutchison, Brandon Norlund, Adam Pyszczynski, Andrew Rodewald, and Eric Thrailkill for their assistance in conducting this research. This work was presented in part at the 38th Annual Convention of the Association for Behavior Analysis International in Seattle, May 2012.

\section{References}

Ahearn, W. H., Clark, K. M., Gardenier, N. C., Chung, B., \& Dube, W. V. (2003). Persistence of stereotypic behavior: Examining the effects of external reinforcers. Journal of Applied Behavior Analysis, 36, 439448.

Cohen, S. L. (1996). Behavioral momentum of typing behavior in college students. Journal of Behavior Analysis and Therapy, 1, 36-51.

da Silva, S. P., Maxwell, M. E., \& Lattal, K. A. (2008). Concurrent resurgence and behavioral history. Journal of the Experimental Analysis of Behavior, 90, 313-331.

Higgins, S. T., Bernstein, I. M., Washio, Y., Heil, S. H., Badger, G. J., Skelly, J. M., \& Solomon, L. J. (2010). Effects of smoking cessation with voucher-based contingency management on birth outcomes. Addiction, 105, 2023-2030.

Igaki, T., \& Sakagami, T. (2004). Resistance to change in goldfish. Behavioural Processes, 66, 139-152.

Lattal, K. A., \& St. Peter Pipkin, C. (2009). Resurgence of previously reinforced responding: Research and application. Behavior Analyst Today, 10, 254-265.

Leitenberg, H., Rawson, R. A., \& Mulick, J. A. (1975). Extinction and reinforcement of alternative behavior. Journal of Comparative and Physiological Psychology, 88, 640-652.
Lieving, G. A., \& Lattal, K. A. (2003). Recency, repeatability, and reinforcer retrenchment: An experimental analysis of resurgence. Journal of the Experimental Analysis of Behavior, 80, 217-233.

Mace, F. C., McComas, J. J., Mauro, B. C., Progar, P. R., Taylor, B., Ervin, R., \& Zangrillo, A. N. (2010). Differential reinforcement of alternative behavior increases resistance to extinction: Clinical demonstration, animal modeling, and clinical test of one solution. Journal of the Experimental Analysis of Behavior, 93, 349-367.

Nevin, J. A., \& Grace, R. C. (2000). Behavioral momentum and the law of effect. Behavioral and Brain Sciences, 23, 73-130.

Nevin, J. A., \& Grace, R. C. (2005). Resistance to extinction in the steady state and in transition. Journal of Experimental Psychology: Animal Behavior Processes, 31, 199-212. doi:10.1037/0097-7403.31.2.199

Nevin, J. A., Mandell, C., \& Atak, J. R. (1983). The analysis of behavioral momentum. Journal of the Experimental Analysis of Behavior, 39, 49-59.

Nevin, J. A., McLean, A. P., \& Grace, R. C. (2001). Resistance to extinction: Contingency termination and generalization decrement. Animal Learning \& Behavior, 29, 176-191. doi:10.3758/BF03192826

Nevin, J. A., \& Shahan, T. A. (2011). Behavioral momentum theory: Equations and applications. Journal of Applied Behavior Analysis, 44, 877-895. doi:10.1901/jaba.2011.44-877

Nevin, J. A., Tota, M. E., Torquato, R. D., \& Shull, R. L. (1990). Alternative reinforcement increases resistance to change: Pavlovian or operant contingencies? Journal of the Experimental Analysis of Behavior, 53, 359-379.

Petscher, E. S., Rey, C., \& Bailey, J. S. (2009). A review of empirical support for differential reinforcement of alternative behavior. Research in Developmental Disabilities, 30, 409-425.

Podlesnik, C. A., \& Shahan, T. A. (2010). Extinction, relapse, and behavioral momentum. Behavioural Processes, 84, 400-411.

Quick, S. L., Pyszczynski, A. D., Colston, K. A., \& Shahan, T. A. (2011). Loss of alternative non-drug reinforcement induces relapse of cocaine-seeking in rats: Role of dopamine $D_{1}$ receptors. Neuropsychopharmacology, 36, 1015-1020. doi:10.1038/npp. 2010.239

Shahan, T. A., \& Burke, K. A. (2004). Ethanol-maintained responding of rats is more resistant to change in a context with added non-drug reinforcement. Behavioral Pharmacology, 15, 279-285.

Shahan, T. A., \& Sweeney, M. M. (2011). A model of resurgence based on behavioral momentum theory. Journal of the Experimental Analysis of Behavior, 95, 91-108. doi:10.1901/jeab.2011.95-91

Silverman, K., Wong, C. J., Needham, M., Diemer, K. N., Knealing, T., Crone-Todd, D., \& Kolodner, K. (2007). A randomized trial of employment-based reinforcement of cocaine abstinence in injection drug users. Journal of Applied Behavior Analysis, 40, $387-$ 410. doi:10.1901/jaba.2007.40-387

Volkert, V. M., Lerman, D. C., Call, N. A., \& Trosclair-Lasserre, N. (2009). An evaluation of resurgence during treatment with functional communication training. Journal of Applied Behavior Analysis, 42, 145-160.

Wacker, D. P., Harding, J. W., Berg, W. K., Lee, J. F., Schieltz, K. M., Padilla, Y. C., \& Shahan, T. A. (2011). An evaluation of persistence of treatment effects during long-term treatment of destructive behavior. Journal of the Experimental Analysis of Behavior, 96, 261282.

Winterbauer, N. E., \& Bouton, M. E. (2010). Mechanisms of resurgence of an extinguished instrumental behavior. Journal of Experimental Psychology: Animal Behavior Processes, 3, 343-353. doi:10. 1037/a0028853

Winterbauer, N. E., Lucke, S., \& Bouton, M. E. (2013). Some factors modulating the strength of resurgence after extinction of an instrumental behavior. Learning and Motivation, 44, 60-71. doi:10. 1016/j.1mot.2012.03.003 